\title{
Overdiagnosis and Overtreatment of Malaria in Children That Presented with Fever in Lagos, Nigeria
}

\author{
Oladipo O. Oladosu, ${ }^{1}$ and Wellington A. Oyibo ${ }^{1,2}$ \\ ${ }^{1}$ ANDI Centre of Excellence for Malaria Diagnosis and College of Medicine, University of Lagos, Idi-Araba, Lagos, Nigeria \\ ${ }^{2}$ ANDI Centre of Excellence for Malaria Diagnosis, International Malaria Microscopy Training and RDT QA Programme, \\ WHO/TDR/FIND Malaria Specimen Bank Site, and Department of Medical Microbiology and Parasitology, College of Medicine, \\ University of Lagos, Idi-Araba, Lagos, Nigeria
}

Correspondence should be addressed to Wellington A. Oyibo; wellao@yahoo.com

Received 5 April 2012; Accepted 17 May 2012

Academic Editors: D. Bachani, T. Hazir, M. Ramharter, F. Remoue, and E. Seminari

Copyright ( 2013 O. O. Oladosu and W. A. Oyibo. This is an open access article distributed under the Creative Commons Attribution License, which permits unrestricted use, distribution, and reproduction in any medium, provided the original work is properly cited.

\begin{abstract}
Background. Malaria diagnosis has been largely done clinically. The implication is the likely overdiagnosis of malaria when diagnosis is done soley on the basis of symptoms. Methods. Parasitological diagnosis was done among 1211, 0-12 years old febrile children that attended a Primary Health Centre in Lagos, Nigeria, who were diagnosed clinically and treated based on symptoms. Results. A total of 251 of $1,211(20.7 \%)$ children less than 12 years old and 174 of the 1,027 of children $0-\leq 5$ yrs (16.9\%) were slide positive while $853(83.1 \%)$ of $0-\leq 5 \mathrm{yrs}$ that were slide negative were treated with Artemisinin based combination therapies (ACTs) in line with the Integrated Management of Childhood Infectioins (IMCI) guidelines and standard practice of the Clinic. Chills, diarrhoea, convulsions, headache, cough, respiratory distress, inactivity, loss of apetite, and vomiting occured significantly in the $0-\leq 5$ and $>5-12$ years old malaria negative children. Conclusions. Overdiagnosis and overtreatment of malaria in this study was high. Therefore, malaria medicines should be prescribed on the basis of parasitological confirmation of all suspected malaria cases. The availability of quality-assured malaria rapid diagnostic tests (RDTs) is a useful tool to confirm malaria cases while the cause of the non-malaria fevers can be followed up and managed appropriately.
\end{abstract}

\section{Introduction}

In areas of intense malaria transmission, such as large parts of tropical Africa, where the burden of malaria is greatest and where severe disease and mortality are largely confined to children under 5 years of age, malaria treatment is often dispensed on the basis of "fever" and other malariaassociated symptoms such as chills, headache, vomiting, respiratory distress and so forth, rather than on the basis of a parasitologically confirmed diagnosis. This is also mainly due to the wholesale implementation of the Integrated Management of Childhood Illness guidelines [1]. One of the reasons for presumptive treatment is the fear of rapid mortality of untreated malaria, especially in young children. Until recently, the diagnosis of malaria in children in most African setting was on the clinical basis of fever and other malaria related symptoms that are, however, not specific to malaria alone.

The clinical presentation of malaria is highly variable and overlaps with that of a number of other common illnesses, including pneumonia, which are associated with significant morbidity and mortality [2-4]. The policy of presumptive treatment of malaria for all febrile illnesses has been widely advocated in sub-Saharan Africa, especially in young children $[5,6]$. Attempts to develop clinical scoring systems with high predictive values have largely been unsuccessful [7], and health workers without access to tools for parasite-based diagnosis often manage most or all suspected malaria cases as malaria. This practice continues to be included in medical training and in national treatment guidelines [8].

Presumptive management of fevers and/or other suspected symptoms of malaria as malaria results in significant 
overdiagnosis and overtreatment, even in high-risk areas. In many settings, especially where malaria is seasonal or where intensive disease control efforts are implemented, a small minority of febrile patients may be parasitaemic $[9,10]$.

A history of fever and positive blood smear on light microscopy is the standard for malaria diagnosis and basis of treatment, but in practice this is not often adhered to. Microscopy is often not used even when it is available [11].

Malaria control measures have been scaled up in malaria endemic countries and there is growing evidence of overtreatment of malaria as fewer cases of fever or suspected cases of malaria are likely to be malaria upon confirmation $[9,11,12]$. Over-prescription of ACT may result in substantial unnecessary use of this class of drug and the risk of developing resistance. In addition, blind treatment of malaria without parasitological confirmation of the parasite deviates from best practices. At present, episodes of malarial fever among children under five are reported to be in decline in Africa [12], and because of this, the World Health Organization (WHO) recommends that malaria treatment should begin with parasitological diagnosis for all age groups [13-15]. Light microscopy or malaria rapid diagnostic tests (RDTs) offer an inexpensive and practical means of improving malaria diagnosis and treatment in areas of low transmission $[11,16]$.

With reports of the decline in incidence of malaria [1719] and with almost universal introduction of artemisininbased combination therapy (ACT), it is imperative that parasite-based confirmation of malaria be scaled up even in the under fives, given the proclivity to overtreat malaria due to clinical overdiagnosis. Providing the evidence of overdiagnosis of malaria in children less than five years is indispensable in the implementation of the World Health Organization's and the National Malaria Diagnosis and Treatment policy on universal malaria testing for all age groups suspected to have malaria. This paper reports the parasitological confirmation of malaria in children that presented with fever or history of fever in the last 24 hours in Lagos, Nigeria, who received malaria medicines on the basis of clinical presentations in accordance with the IMCI guideline.

\section{Materials and Methods}

2.1. Study Area. This study was conducted at the St. Kizito Primary Health Centre, Lekki-Lagos, Southwestern Nigeria-a holoendemic area for malaria. The St. Kizito Primary Health Centre, Lekki, receives patients from localities with environmental conditions which most likely contribute to human-mosquito contact and which may invariably influence urban malaria transmission.

This is a descriptive study among children less than 12 years old that presented with fever or history of fever between July 2007 and March 2008. Malaria microscopy was done for all children who had been presumptively diagnosed and antimalarial medicines prescribed in accordance with the IMCI guidelines.
2.2. Study Population. A total of 1,211 children, aged $0-12$ years, who presented with fever or history of fever in the last 24 hours at the Outpatient's Department (OPD) were recruited into this study. The children were enrolled if they met the following inclusion criteria: 0 to 12 years, documented fever at presentation or history of fever in the last 24 hours, absence of danger signs of complicated/severe malaria and known serious chronic disease, and willingness of the parent/guardian to provide written assent and consent. Those that presented with signs of complicated malaria namely convulsions/coma, prostration, severe vomiting, and so forth were excluded and these were managed appropriately or referred. The case report form (CRF) that included documented axillary body temperature, history of fever, and other presenting symptoms was completed for each patient before making a finger prick for malaria blood smear preparation. All the children that participated were treated according to the standard routine care of the health facility and this was based on the IMCI guidelines. The results of the malaria microscopy tests were made available to the management of the health facility as a basis for the review of their standard malaria case-management protocol. Good Clinical Laboratory Practice guidelines were adhered to in the study.

2.3. Parasitological Diagnosis of Malaria Parasitaemia. Immediately after clinical examination by the managing physician, each child that has been handed malaria medicines was asked to undertake the malaria microscopy test. Thick and thin smears were made on the same slide for each child from finger prick using a sterile lancet. Two slides were made for each child. The first slide was the read " $(\mathrm{R})$ " slide (that is the slide that was read), while the other slide was archived "(A)" slide. This is in line with the quality assurance process of the ANDI Centre of Excellence for Malaria Diagnosis, College of Medicine, University of Lagos, Lagos, Nigeria. The thin films were fixed with methanol and left to dry. The prepared smears were stained with $3 \%$ Giemsa at a $\mathrm{pH}$ of 7.2 for 45 minutes. The staining process was quality controlled to ensure that the morphology of the malaria parasites in positive slides were distinct and clear.

The parasite density was computed as the number of parasites per 500 leucocytes on a thick film and reported as parasites per microlitre of blood assuming an average white blood-cell count of 8000 per $\mu \mathrm{L}$ [20]. Stained slides were examined under the light microscope using $\times 100$ objective lens (immersion oil). A slide was considered negative after 100 high power fields (HPF) have been examined. Another Microscopist, the second reader, was made to reread each slide. Parasite counts of $>20 \%$ discordance between two readers were reread by a third reader, who served as the tie breaker. However, parasite counts of less than 20\% discordance between the first and second readers were accepted and the mean parasite count taken to compute the parasite density or parasitaemia for each child. The Microscopists that read the slides were prequalified before the study through a rigorous process. 
TABLE 1: Prevalence and intensity of Plasmodium in the different age groups.

\begin{tabular}{lcccc}
\hline Age $(\mathrm{yrs})$ & No examined & No positive $(\%)$ & Parasitaemia range & Mean parasitaemia $(\mathrm{p} / \mu \mathrm{L})$ \\
\hline $0-\leq 5$ yrs & 1,027 & $174(16.9)$ & $15-678,269$ & $39,903.6$ \\
$>5-12$ yrs & 183 & $77(42.1)$ & $16-432,077$ & $40,678.2$ \\
$0-12$ yrs & 1,211 & $251(20.7)$ & $15-678,269$ & $40,141.2$ \\
$P$ & & $<0.001$ & & $<0.001$ \\
\hline
\end{tabular}

TABLE 2: Symptomatic presentation in children $0-\leq 5$ and $>5-12$ years with or without Malaria Parasites (MP).

\begin{tabular}{|c|c|c|c|c|}
\hline Symptoms & $\begin{array}{l}\text { MP positive } \\
0-\leq 5 \text { yrs (\%) }\end{array}$ & $\begin{array}{l}\text { MP negative } \\
0-\leq 5 \text { yrs (\%) }\end{array}$ & $\begin{array}{c}\text { MP positive } \\
>5-12 \text { yrs (\%) }\end{array}$ & $\begin{array}{l}\text { MP negative } \\
>5-12 \text { yrs }(\%)\end{array}$ \\
\hline Chills & $38(21.8)$ & $136(78.2)$ & $16(20.8)$ & $61(79.2)$ \\
\hline Convulsion & $1(0.6)$ & $173(99.4)$ & $1(1.3)$ & $76(98.7)$ \\
\hline Cough & $57(32.8)$ & $117(67.2)$ & $22(28.6)$ & $55(71.4)$ \\
\hline Diarrhoea & $26(14.9)$ & $148(85.1)$ & $1(1.3)$ & $76(98.7)$ \\
\hline Fever $\left(\geq 37.5^{\circ} \mathrm{C}\right)$ & $159(91.4)$ & $15(8.6)$ & $69(89.6)$ & $8(10.4)$ \\
\hline Headache & $19(10.9)$ & $155(89.1)$ & $33(42.9)$ & $44(57.1)$ \\
\hline Inactivity & $1(0.6)$ & $174(99.4)$ & $0(0)$ & $77(100)$ \\
\hline Loss of appetite & $61(35.1)$ & $113(64.9)$ & $22(28.6)$ & $55(71.4)$ \\
\hline Respiratory distress & $6(3.4)$ & $168(96.6)$ & $3(3.9)$ & $74(96.1)$ \\
\hline Vomiting & $45(25.9)$ & $129(74.1)$ & $24(31.2)$ & $53(68.8)$ \\
\hline
\end{tabular}

2.4. Ethical Approval. Ethical approval was obtained from the Research, Grants and Experimentation Committee, College of Medicine, University of Lagos, Nigeria. Assent was obtained from the children with corresponding consent from their parents/guardian before they were enrolled. All patients studied received the appropriate standard of care.

2.5. Data Analysis. The Epi-info v.3.2.2 (CDC, Atlanta, Georgia, USA) was used both for data entry and analysis. Proportions were compared by Pearson's Chi-square or Fisher's exact tests. The children were grouped into age $0-\leq 5$ years and $>5-12$ years for the purpose of data analysis.

\section{Results}

A total of 1,211 children, 0-12 years old, were enrolled in the study. The children tested included 658(54.4\%) males and $553,(45.6 \%)$ females. The mean age \pm SD of the children was $2.65 \pm 2.83$ while their mean body temperature was $37.8^{\circ} \mathrm{C}$ (range, $35.5-42^{\circ} \mathrm{C}$ ).

Out of the total children ( $<12$ years) tested microscopically, $251(20.7 \%)$ were positive for malaria parasites, while the others were slide negative (960 (79.3\%)). Among the $0-\leq 5$ years old children (1028) tested microscopically, 174 (16.9\%) were positive for malaria parasite while others were slide negative $(854(83.1 \%))(P=0.001)$. The $>5-12$-years-old children had a prevalence of $42.1 \%$ (Table 1 ). All the children between $0-\leq 5$ years received antimalarials in compliance with the IMCI guidelines and the standard routine practice of the clinic. This gave an overtreatment of $83.1 \%$ in children $0-\leq 5$ years based on blood smear microscopy confirmation. The malaria parasite density ranged from 15 parasite $/ \mu \mathrm{L}$ of blood to 678,269 parasites $/ \mu \mathrm{L}$ of blood while the mean parasite density was 39,903.6 parasite/ $\mu \mathrm{L}$ of blood (Table 1 ). The proportion of children with parasite density of 1-500 parasite $/ \mu \mathrm{L}$ of blood among the malaria-positive $0-\leq 5$-year children was $33.9 \%$; 501-1,000 (6.8\%); 1,001-10,000 (22.3\%); $10,001(32.3 \%)$, while $4.8 \%$ had parasite density $>250,000$ parasite $/ \mu \mathrm{L}$ of blood $(P=0.001)$.

Documented fever (measured axillary temperature $\geq 37.5^{\circ} \mathrm{C}$ ) was seen more in the malaria-positive than the malaria-negative children $(0-\leq 5$-years and $>5-12$-years-old children). Chills, diarrhoea, convulsions, headache, cough, respiratory distress, inactivity, loss of appetite, and vomiting occurred significantly in the $0-\leq 5-$ and $>5-12$-year-old malaria-negative children (MP negative) $(P<0.05)$ (Table 2 ). The association of documented fever with positive malaria smear was significant $(P<0.05)$.

Plasmodium falciparum was the dominant species encountered (96.5\%). This was followed by Plasmodium malariae (1.2\%) and Plasmodium ovale (0.4\%). Mixed infections between Plasmodium falciparum and Plasmodium malariae was $1.2 \%$.

\section{Discussion}

Malaria is a problematic health issue in children, but it is frequently overdiagnosed and results in failure to treat other life-threatening conditions. This study highlighted the overdiagnosis and overtreatment of malaria in Lagos, Nigeria, a country in the Sub-Saharan region of Africa where $95 \%$ of malaria cases are due to Plasmodium falciparum. In children under the age of five years, only $16.9 \%$ had malaria positive slides and $P$. falciparum was the most predominant species. The prevalence of $P$. falciparum malaria in our study was higher than 12\% reported in Tanzania [21] and lower than $56.9 \%$ reported in a similar study in Nigeria [22]. Presumptive 
treatment of all childhood fevers or suspected malaria cases as malaria results in malaria overdiagnosis, which means other causes of febrile illnesses, such as pneumonia and meningitis, are missed at initial presentation at health facilities until a much later time when the child's condition does not improve. Indirect evidence shows that the overdiagnosis of malaria contributes to increasing ill health, death, loss of productivity, and a vicious cycle of deepening poverty in the most vulnerable sections of society [23]. Treating all childhood malaria-associated presentations on the basis of clinical suspicion as malaria means that poor people are wasting valuable resources on malaria drugs and failing to be treated for other potentially life-threatening illnesses [23]. Also, the indiscriminate use of ACTs potentially increases the risk of an emergence of resistance [24, 25].

The presumptive diagnosis of malaria previously advocated for malaria endemic areas may have always resulted in some degree of overtreatment especially in under fives, given poor malaria microscopy method and slow evaluation of the IMCI guidelines in respect to malaria in several settings. Considering the various reports of the decline in malaria prevalence from several parts of sub-Saharan Africa [19, 26-28], continued use of clinical presentations as a basis for treatment is likely to increase the degree of overtreatment and inevitably failure to treat the alternative causes of fever and this could lead to high morbidity and mortality. In a study where presumptive diagnosis of malaria was used as an indicator for malaria diagnosis in children, only $24.8 \%$ of the patients had a malaria episode according to the case definition, resulting in more than $75 \%$ of the patients receiving antimalarial drugs unnecessarily [29]. History of fever was commonly reported but was an unreliable indicator being neither associated with presence of parasitaemia nor with fulfilling the malaria case definition [29].

Malaria has very similar clinical presentations to other infections, making it difficult to clinically differentiate from conditions such as typhoid, pneumonia, and other bacteraemia [30]. Clinical diagnosis of malaria, especially in infants, has a poor diagnostic accuracy and a low positive predictive value because symptoms and signs are variable and can easily be mimicked by other infectious and noninfectious diseases [31]. Fever was shown to be the main clinical presentation for malaria in endemic areas [32] and this contradicts with reports of a study in The Gambia [33] where fever or history of fever resulted in the overdiagnosis of malaria. Diarrhoea, chills, convulsion, cough, inactivity, vomiting, and headache were often used by health workers as a basis for treating malaria. However, these were not associated with malaria in this study. Some earlier reports on other common presenting symptoms in children showed that malaria episodes were associated with diarrhoea [34] and headache [35], chills and arthralgias [36].

The result of this study is consistent with a study in Zambia [37] where children were overtreated with Artemisinin based combination therapy. Furthermore, overtreatment of malaria was reported from several parts of East Africa, mainly in areas with low to moderate or high perennial transmission [29, 38-40]. Significantly, antimalarials were prescribed for
$58.4 \%$ of patients with negative blood smear results in Zambia [41]. All the less-than-five-year-old children with slidenegative microscopy results in this study (83.1\%) were treated with antimalarials (artemether-lumefantrine) according to the IMCI guidelines.

Our findings highlight the need for the scaling-up of parasitological confirmation of all patients suspected to have malaria in Lagos, Nigeria, without delay. It provides evidence of gross overdiagnosis of malaria and overtreatment with the ACTs. Ironically, when it is an overdiagnosis of malaria, it is a poor diagnosis and a likely poor management of the presenting conditions that delays the institution of prompt and effective management. The availability of quality-assured malaria rapid diagnostic tests (RDTs) raises hope for prompt and effective malaria case management.

Therefore, improving the diagnostic system (microscopy and rapid diagnostic tests, health workers training, infrastructure for diagnostics, quality assurance for diagnostic tools, etc.) is critical for the accurate diagnosis of malaria. Malaria RDTs can quickly confirm the absence or presence of malaria. The available tools should therefore be used in the case management of malaria in children. The implementation of the WHO's universal parasitological confirmation of malaria as adapted by most African countries creates an opportunity to improve health delivery services in a more positive way by instituting evidence-based practices. This study therefore negates the arguments to continue with presumptive malaria case management practices. The IMCI guideline that recommends presumptive treatment of malaria in children under five years of age should be modified appropriately to include parasitological confirmation of malaria where the tools are available. Access to prompt and quality diagnostics and behavioural change communication among health workers should be strengthened to scale up parasitological confirmation of malaria.

\section{Conflict of Interests}

The authors declare they have no Conflict of Interests.

\section{Acknowledgments}

The authors thank WHO/TDR, the Foundation for Innovative New Diagnostics (FIND), and other partners that provided the platform to improve their malaria microscopy diagnostics capacity through the prequalification/training of their malaria microscopists. Furthermore, The authors thank the guardians/parents for giving accent/consent of their wards to participate in this study, it is appreciated. Finally, The authors thank Dr. Chiara Meziliarra and Mrs Peju Hammeed and the management and Staff of St. Kizito Clinic for the permission to use the health facility.

\section{References}

[1] World Health Organization, Technical Updates of the Guidelines on the Integrated Management of Childhood Illness (IMCI), 2005. 
[2] M. English, J. Berkley, I. Mwangi et al., "Hypothetical performance of syndrome-based management of acute paediatric admissions of children aged more than 60 days in a Kenyan district hospital," Bulletin of the World Health Organization, vol. 81, no. 3, pp. 166-173, 2003.

[3] K. Källander, J. Nsungwa-Sabiiti, and S. Peterson, "Symptom overlap for malaria and pneumonia-policy implications for home management strategies," Acta Tropica, vol. 90, no. 2, pp. 211-214, 2004.

[4] J. A. Berkley, K. Maitland, I. Mwangi et al., "Use of clinical syndromes to target antibiotic prescribing in seriously ill children in malaria endemic area: observational study," British Medical Journal, vol. 330, no. 7498, pp. 995-999, 2005.

[5] R. D. Gosling, C. J. Drakeley, A. Mwita, and D. Chandramohan, "Presumptive treatment of fever cases as malaria: help or hindrance for malaria control?" Malaria Journal, vol. 7, article 132, 2008.

[6] M. English, H. Reyburn, C. Goodman, and R. W. Snow, "Abandoning presumptive antimalarial treatment for febrile children aged less than five years-a case of running before we can walk?" PLoS Medicine, vol. 6, no. 1, Article ID e1000015, 2009.

[7] T. W. Mwangi, M. Mohammed, H. Dayo, R. W. Snow, and K. Marsh, "Clinical algorithms for malaria diagnosis lack utility among people of different age groups," Tropical Medicine and International Health, vol. 10, no. 6, pp. 530-536, 2005.

[8] M. Amexo, R. Tolhurst, G. Barnish, and I. Bates, "Malaria misdiagnosis: effects on the poor and vulnerable," The Lancet, vol. 364, no. 9448, pp. 1896-1898, 2004.

[9] H. Reyburn, H. Mbakilwa, R. Mwangi et al., "Rapid diagnostic tests compared with malaria microscopy for guiding outpatient treatment of febrile illness in Tanzania: randomised trial," British Medical Journal, vol. 334, no. 7590, pp. 403-406, 2007.

[10] World Health Organization, "Roll back malaria," World Malaria Report WHO/HTM/GMP/2008.1.

[11] H. Reyburn, J. Ruanda, O. Mwerinde, and C. Drakeley, “The contribution of microscopy to targeting antimalarial treatment in a low transmission area of Tanzania," Malaria Journal, vol. 5, article 4, 2006.

[12] V. D'Acremont, C. Lengeler, and B. Genton, "Reduction in the proportion of fevers associated with Plasmodium falciparum parasitaemia in Africa: a systematic review," Malaria Journal, vol. 9, no. 1, article 240, 2010.

[13] World Health Organization, Guidelines for the Treatment of Malaria, World Health Organization, Geneva, Switzerland, 2nd edition, 2010.

[14] J. Zarocostas, "Malaria treatment should begin with parasitological diagnosis where possible, says WHO," British Medical Journal, vol. 340, Article ID c1402, 2010.

[15] B. Graz, M. Willcox, T. Szeless, and A. Rougemont, “Test and treat or presumptive treatment for malaria in high transmission situations? A reflection on the latest WHO guidelines," Malaria Journal, vol. 10, article 136, 2011.

[16] G. J. Bastiaens, E. Schaftenaar, A. Ndaro, M. Keuter, T. Bousema, and S. A. Shekalaghe, "Malaria diagnostic testing and treatment practices in three different Plasmodium falciparum transmission settings in Tanzania: before and after a government policy change," Malaria Journal, vol. 10, article 76, 2011.

[17] M. K. Bouyou-Akotet, D. P. Mawili-Mboumba, E. Kendjo et al., "Evidence of decline of malaria in the general hospital of Libreville, Gabon from 2000 to 2008," Malaria Journal, vol. 8, no. 1 , article $300,2009$.
[18] C. Delacollette, C. D’Souza, E. Christophel et al., "Malaria trends and challenges in the greater mekong subregion," Southeast Asian Journal of Tropical Medicine and Public Health, vol. 40, no. 4, pp. 674-691, 2009.

[19] J. Satoguina, B. Walther, C. Drakeley et al., "Comparison of surveillance methods applied to a situation of low malaria prevalence at rural sites in the Gambia and Guinea Bissau," Malaria Journal, vol. 8, no. 1, article 274, 2009.

[20] B. M. Greenwood and J. R. M. Armstrong, "Comparison of two simple methods for determining malaria parasite density," Transactions of the Royal Society of Tropical Medicine and Hygiene, vol. 85, no. 2, pp. 186-188, 1991.

[21] H. D. Mazigo, M. Wilfred, E. E. Ambrose, B. R. Kidenya, and E. J. Kweka, "Confirmed malaria cases among children under five with fever and history of fever in rural Tanzania," BMC Research Notes, vol. 4, article 359, 2011.

[22] E. I. Ikeh and N. N. Teclaire, "Prevalence of malaria parasitaemia and associated factors in febrile under- 5 children seen in primary health care centres in Jos, North Central Nigeria," The Nigerian Postgraduate Medical Journal, vol. 15, no. 2, pp. 65-69, 2008.

[23] MKP Policy Brief, "Improving the quality of malaria diagnosis and laboratory services in resource-poor countries," 2005, http://www.liv.ac.uk/lstm/ majorprogs/malaria/outputs.htm.

[24] G. O. Gbotosho, C. T. Happi, A. Ganiyu, O. A. Ogundahunsi, A. Sowunmi, and A. M. Oduola, "Potential contribution of prescription practices to the emergence and spread of chloroquine resistance in south-West Nigeria: caution in the use of artemisinin combination therapy," Malaria Journal, vol. 8, no. 1, article 313, 2009.

[25] S. Yeung, D. Socheat, V. S. Moorthy, and A. J. Mills, "Artemisinin resistance on the Thai-Cambodian border," The Lancet, vol. 374, no. 9699, pp. 1418-1419, 2009.

[26] W. P. O’Meara, P. Bejon, T. W. Mwangi et al., "Effect of a fall in malaria transmission on morbidity and mortality in Kilifi, Kenya," The Lancet, vol. 372, no. 9649, pp. 1555-1562, 2008.

[27] A. Rodrigues, J. A. Schellenberg, P. E. Kofoed, P. Aaby, and B. Greenwood, "Changing pattern of malaria in Bissau, Guinea Bissau," Tropical Medicine and International Health, vol. 13, no. 3, pp. 410-417, 2008.

[28] P. De Beaudrap, C. Nabasumba, F. Grandesso et al., "Heterogeneous decrease in malaria prevalence in children over a sixyear period in South-Western Uganda," Malaria Journal, vol. 10, article 132, 2011.

[29] R. Ndyomugyenyi, P. Magnussen, and S. Clarke, "Diagnosis and treatment of malaria in peripheral health facilities in Uganda: findings from an area of low transmission in South-Western Uganda," Malaria Journal, vol. 6, article 39, 2007.

[30] S. C. Redd, P. B. Bloland, P. N. Kazembe, E. Patrick, R. Tembenu, and C. C. Campbell, "Usefulness of clinical casedefinitions in guiding therapy for African children with malaria or pneumonia," The Lancet, vol. 340, no. 8828, pp. 1140-1143, 1992.

[31] B. Hogh, N. T. Marbiah, P. A. Burghaus, and P. K. Andersen, "Relationship between maternally derived anti-Plasmodium falciparum antibodies and risk of infection and disease in infants living in an area of Liberia, West Africa, in which malaria is highly endemic," Infection and Immunity, vol. 63, no. 10, pp. 4034-4038, 1995.

[32] I. T. Runsewe-Abiodun, O. B. Ogunfowora, and B. M. Fetuga, "Neonatal malaria in Nigeria-a 2 year review," BMC Pediatrics, vol. 6, article 19, 2006. 
[33] B. O. Olaleye, L. A. Williams, U. D’Alessandro et al., "Clinical predictors of malaria in Gambian children with fever or a history of fever," Transactions of the Royal Society of Tropical Medicine and Hygiene, vol. 92, no. 3, pp. 300-304, 1998.

[34] O. Müller, C. Traoré, H. Becher, and B. Kouyaté, "Malaria morbidity, treatment-seeking behaviour, and mortality in a cohort of young children in rural Burkina Faso," Tropical Medicine and International Health, vol. 8, no. 4, pp. 290-296, 2003.

[35] S. E. Clarke, S. Brooker, J. K. Njagi et al., "Malaria morbidity among school children living in two areas of contrasting transmission in Western Kenya," American Journal of Tropical Medicine and Hygiene, vol. 71, no. 6, pp. 732-738, 2004.

[36] S. D. Fernando and A. R. Wickremasinghe, "The clinical and epidemiological features of childhood malaria in a moderately endemic area of Sri Lanka," Southeast Asian Journal of Tropical Medicine and Public Health, vol. 33, no. 4, pp. 671-677, 2002.

[37] P. Chanda, B. Hamainza, S. Mulenga, V. Chalwe, C. Msiska, and E. Chizema-Kawesha, "Early results of integrated malaria control and implications for the management of fever in underfive children at a peripheral health facility: a case study of Chongwe rural health centre in Zambia," Malaria Journal, vol. 8, no. 1, article 49, 2009.

[38] H. Reyburn, R. Mbatia, C. Drakeley et al., "Overdiagnosis of malaria in patients with severe febrile illness in Tanzania: a prospective study," British Medical Journal, vol. 329, no. 7476, pp. 1212-1215, 2004.

[39] J. Eriksen, G. Tomson, P. Mujinja, M. Y. Warsame, A. Jahn, and L. L. Gustafsson, "Assessing health worker performance in malaria case management of underfives at health facilities in a rural Tanzanian district," Tropical Medicine and International Health, vol. 12, no. 1, pp. 52-61, 2007.

[40] J. U. Okebe, B. Walther, K. Bojang et al., "Prescribing practice for malaria following introduction of artemether-lumefantrine in an urban area with declining endemicity in West Africa," Malaria Journal, vol. 9, no. 1, article 180, 2010.

[41] T. Egwang, "Malaria over-diagnosis and over-treatment in Zambia," May 2007, http://www.aidsmap.com/Healthmonitoring/cat/1655/. 


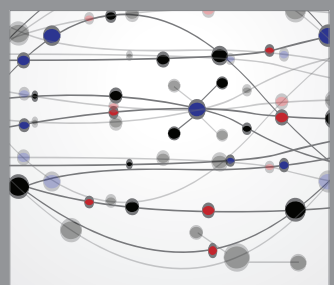

The Scientific World Journal
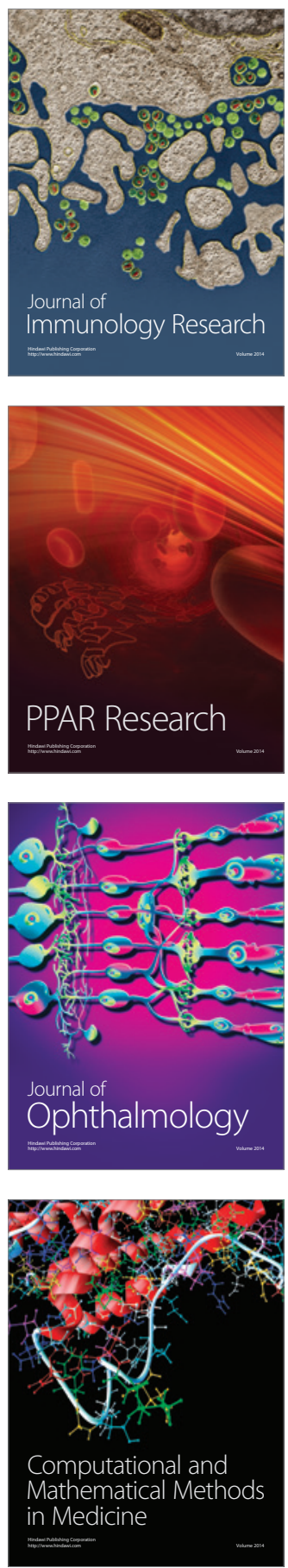

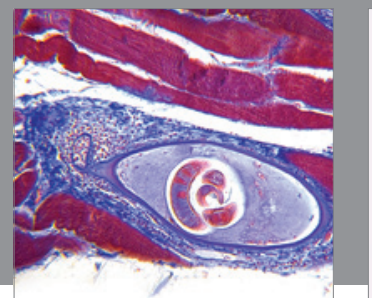

Gastroenterology

Research and Practice
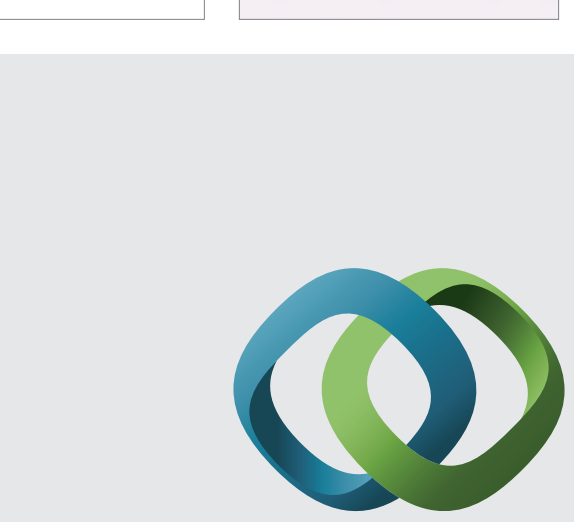

\section{Hindawi}

Submit your manuscripts at

http://www.hindawi.com
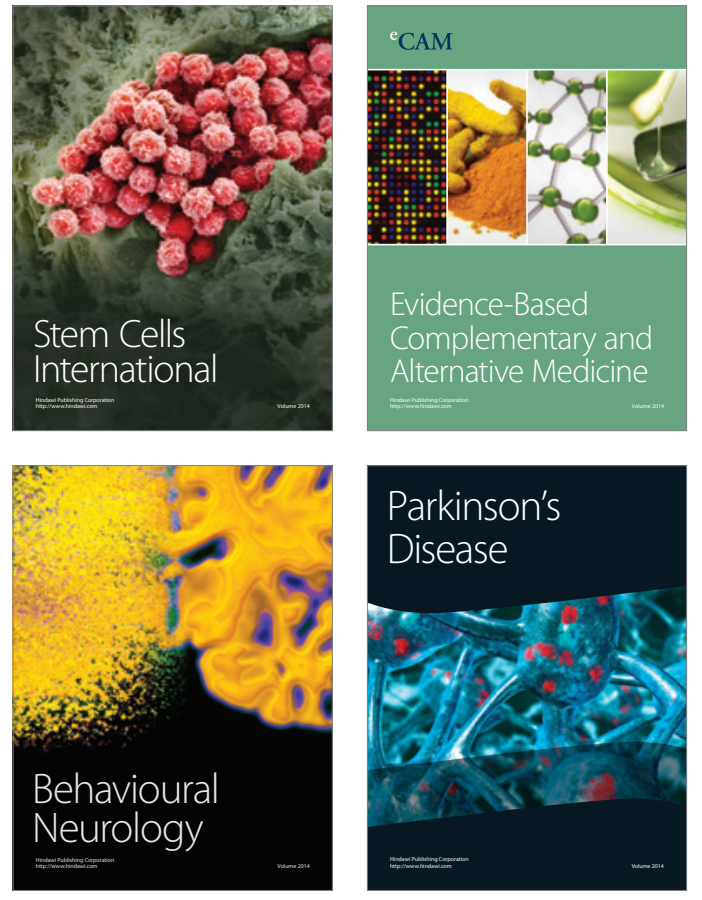
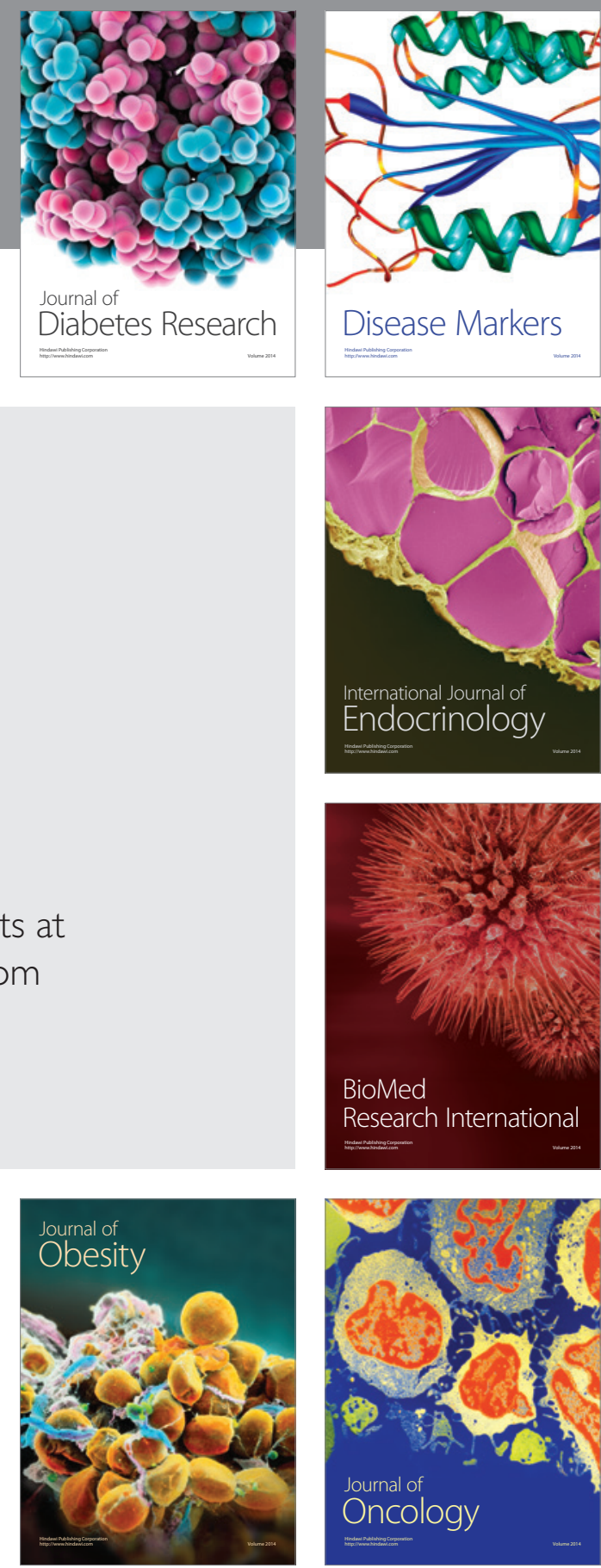

Disease Markers
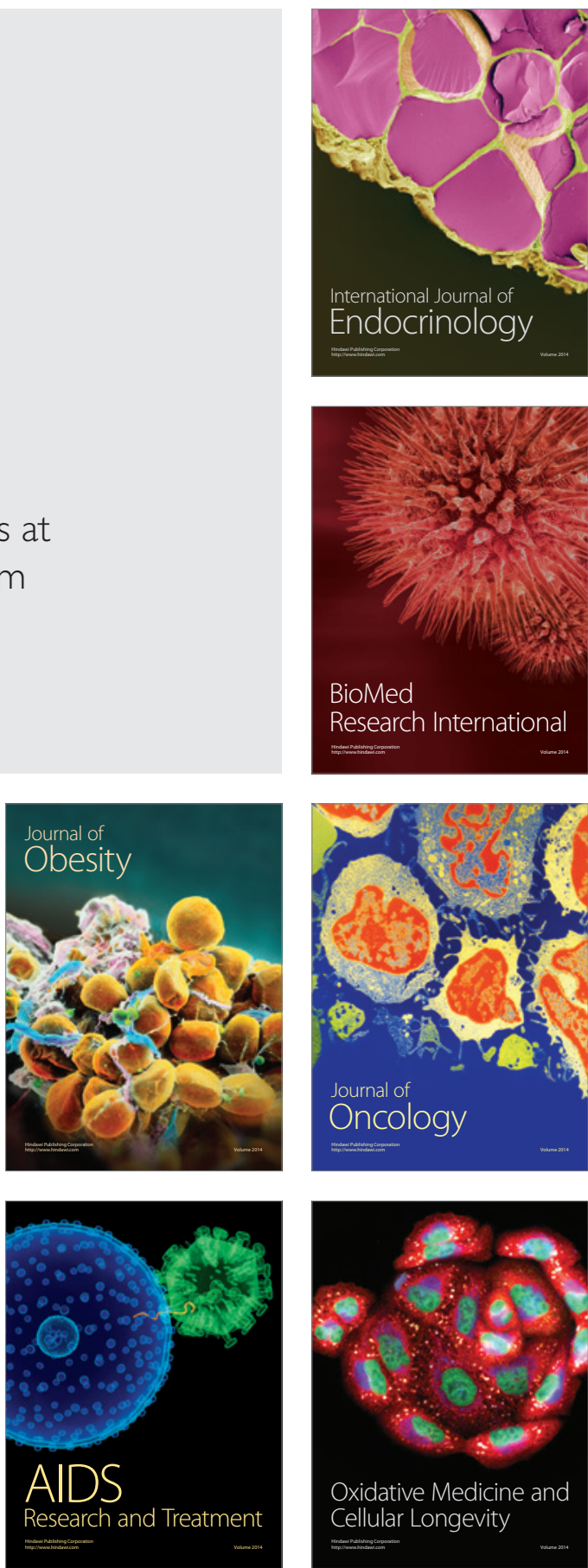